\title{
PERFORMING RELIABLE ABSORBANCE AND FLUORESCENCE MEASUREMENTS WITH LOW BUDGET - A TUTORIAL FOR BEGINNERS
}

\author{
Diandra Nunes Barreto ${ }^{a}$, Vanderli G. Leal ${ }^{a}$, Josiele A. M. Conrado ${ }^{a}$, Gabriel M. Fernandes ${ }^{\text {, }}$ Caio Cesar Souza Machado, \\ Alex D. Batista ${ }^{a}$, Sidnei G. da Silva ${ }^{a}$ and João Flávio da S. Petrucia*, (i) \\ anstituto de Química, Universidade Federal de Uberlândia, Uberlândia - MG, Brasil
}

Recebido em 07/01/2021; aceito em 29/03/2021; publicado na web em 26/04/2021

\begin{abstract}
Molecular absorbance and fluorescence measurements are usually performed by benchtop or portable USB spectrophotometers or fluorometers. However, even the simplest configuration of these instruments requires considerable financial input, which is not affordable to many universities and research groups especially when public politics withdraw funds from science. Light-emitting diodes (LEDs) based devices can be an interesting alternative for low-cost, portable yet reliable measurements of absorbance of fluorescence. Especially if associated with the fabrication of 3D structures by additive manufacturing techniques. Although there are many reviews in the literature dedicated to discussing the theoretical aspects and analytical performance of LED-based devices, we consider that these reviews are not easily applicable for beginners in electronics. It results in the intimidation to employ such powerful tools which could have a huge impact mainly to small and/or initial groups. In this tutorial, we present a guide that will help students and researchers to construct "lab-built" devices based on simple optoelectronic components and 3D printing to perform molecular absorbance and fluorescence measurements for analytical methods. Finally, with this tutorial guide, we anticipate a large spread of the usage of LED devices for reliable analytical measurements that can be extensively used in many scenarios.
\end{abstract}

Keywords: spectrophotometry; spectrofluorimetry; LED; optical methods; instrumentation; 3D printing.

\section{INTRODUCTION}

Absorption and fluorescence are light-induced phenomena widely explored in the field of analytical chemistry research as well as in the related undergraduate and postgraduate teaching topics. ${ }^{1}$ For molecular species, the incidence of light through a sample solution may cause absorption of particular wavelengths by the absorbing molecules leading to electronic transitions from the fundamental ground state $\left(\mathrm{S}_{0}\right)$ to an excited electronic state $\left(\mathrm{S}_{\mathrm{n}}{ }_{\mathrm{n}}\right)$ with finite time (typically 1-10 fentoseconds). This phenomenon is defined as light absorption and it can be followed by a variety of relaxation processes. If the electron relaxes from $S_{1}{ }_{1}$ - with very few exceptions - to the lowest vibrational level (e.g., $S_{1}$ ), via a non-radiative process that usually occurs within $10^{-12} \mathrm{~s}$ or less - and then returns to the ground state $\left(\mathrm{S}_{0}\right)-$ light emission is then generated with lifetimes near of nanoseconds. Thus, the emitted photons that have a different energy than the excitation photons are shifted in frequency. This light emission process is called fluorescence. Several other relaxation pathways compete with the fluorescence emission, including non-radiative processes such as heat, quenching and intersystem crossing. ${ }^{2}$ Figure 1 exemplifies the Jablonski diagram for the Photogem ${ }^{\circledR}$ molecule, a pharmaceutical widely used in Photodynamic Therapy. When UV light reaches the molecule, electronic transitions occur (1), which then return to less energetic levels through internal conversion (2) and light emission (3).
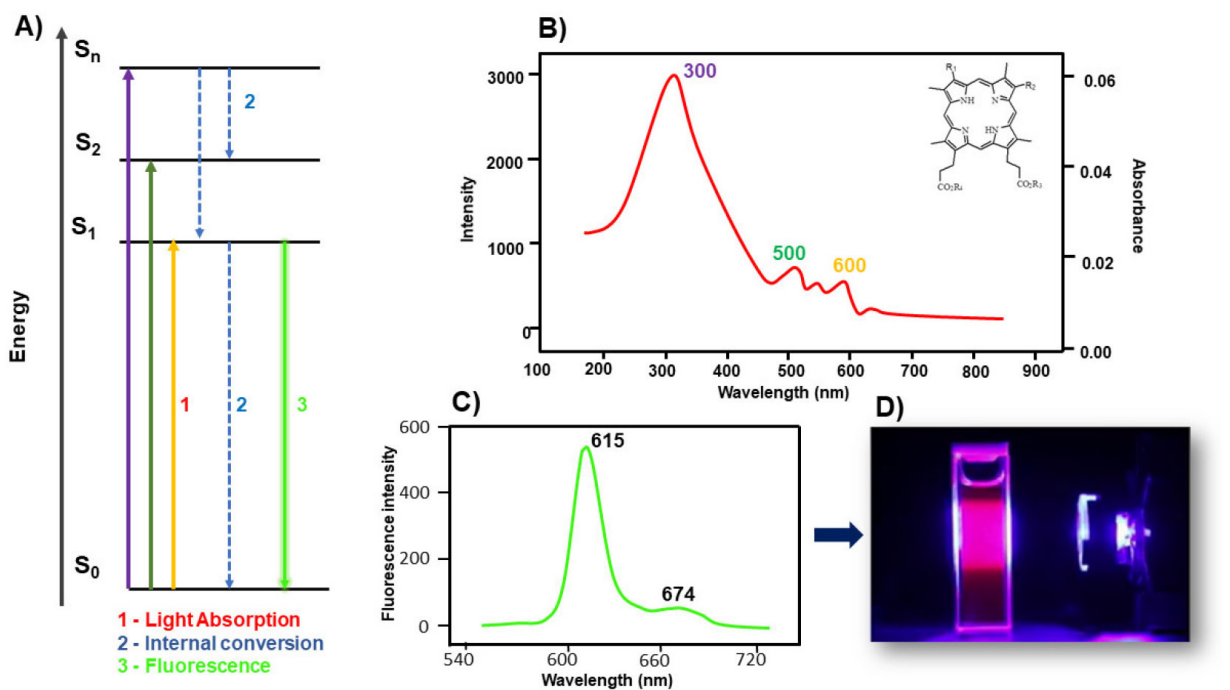

Figure 1. Jablonski diagram (a), Absorption spectrum of the Photogem molecule (b), Emission spectrum of the Photogem molecule (c), Photogem solution under UV light $(d)$ 
Quantitative methods can be based on the measurement of the amount of light absorbed or emitted by using a commercial spectrophotometer or spectrofluorometer., These instruments are composed of a broadband light source, monochromator (e.g. prism or diffraction grade), a sample cell, and a light detector. Such optical instruments have a wide range of applications in the analytical chemistry field as well as for pharmaceutical drug discovery or biotechnological applications. The overall costs of an analysis using absorbance or fluorescence-based techniques are inferior when compared to other optical techniques, such as atomic spectroscopy and near/mid-infrared molecular spectroscopy. However, the acquisition of a conventional spectrophotometer or spectrofluorometer is not feasible for many research groups for several reasons. Fortunately, the market of optoelectronics has boosted up in the last decades resulting in the dropping of the prices of some key-devices which can be used for the development of low-cost yet reliable portable photometers and fluorometers devices.

\section{Fundamentals of light-emitting diodes and photodiodes}

A light-emitting diode (LED) is generally defined as a solidstate semiconductor wherein light emission is generated by a current flow, which is related to electron-hole recombination within the $n-p$ junction of the semiconductor. ${ }^{5}$ The energy related to the distance between valence and conduction bands of the semiconductor will define the wavelength emission of the LED. ${ }^{6}$ The emission spectrum of a light-emitting diode is characterized as a Gaussian curve (as demonstrated in Figure S1) of typically 10 - $100 \mathrm{~nm}$ width and they are widely distributed within the ultraviolet, visible, and infrared electromagnetic spectrum. A LED is optically described by its maximum emission single wavelength and the full width at half maximum (FWHM), which is an indicator of how narrow is the emission band. Ultraviolet and visible LEDs have usually FWHM of 15-20 nm whereas infrared LEDs can reach values higher than $100 \mathrm{~nm}$. Another important LEDs characteristic is the typical current vs voltage curve $(i / \mathrm{V})$ with logarithm behavior as any conventional diode. ${ }^{6}$ Typically, a LED starts to emit light at a specific voltage - defined as forward voltage - where current flow begins. Slight changes in the voltage cause a high variation of current, resulting in changes in the output intensity or even failure of the device. It is important to note that the threshold of the voltage difference is at a range of $0.7-0.8 \mathrm{~V}$.

The employment of LEDs in analytical chemistry started in the 1960 s with the design and construction of a photometer by using a red emitted LED as a light source and a phototransistor as detector. ${ }^{7}$ After a few years, the replacement of the phototransistor by a photodiode was proposed. ${ }^{8}$ Advances on the technology of LEDs allowed the introduction of shorter wavelengths emissions - first with orange and green emissions at the 1970s and blue at the 1990s, resulting in an increase in the number of publications where either a photometer or fluorometer was built by using LED as light source, especially after the introduction of the high brightness blue color LED. ${ }^{9}$ From the 2000s, many studies were developed using stable and long lifetime deep-UV LEDs-based detectors attached to e.g. HPLC, FIA, or CE techniques. ${ }^{10-12}$ Many advantages endorse the high potential of LED in the analytical chemistry field, which are related to the high current efficiency resulting in low heat production providing stable light emission intensity, long lifetime, low cost, narrow light beam, many material package options (e.g. traditional $5 \mathrm{~mm}$ or SMD packages), relative narrow emission bands (compared to conventional lamps) and the possibility of fast switching. Several articles had deeply reviewed the usage of LEDs in the development of analytical devices and are valuable sources of information. ${ }^{6,13-15}$
The replacement of broadband lamps to LEDs as light sources in absorbance/transmittance or fluorescence-based devices requires the employment of detectors for light measurement, which must be equally advantageous as LEDs to keep the simplicity and costs of the final device. In this scenario, the choice for photodiodes (PD) based on semiconductors is usually preferred, as PD as very similar to LED in terms of size, low-cost, and selective to the UV, Vis, or IR region. Besides, PD usually offers stability and low dark current, therefore improving the signal-to-noise ratio. Photodiodes are n-p semiconductors materials where annihilation of charge carriers occurs at the interface, but the absorption of photons releases the charges. When properly operated, the current or voltage generated by light exposition is proportional to light intensity. ${ }^{16}$

Generally, LEDs with emission at the visible spectrum (LEDs-Vis) (i.e. $400-700 \mathrm{~nm}$ ) are much cheaper than LEDs with UV (LEDs-UV) or IR (LEDs-IR) spectral range., Usually, it is possible to find LEDs-Vis in the traditional package (i.e. $5 \mathrm{~mm}$ ) costing less than 0.18 USD, whereas special LEDs-Vis with specific proprieties such as high power, embedded glass lens or mounted LEDs can reach up to 200 USD. The choice for the right LED will depend on each application requirement, but usually, a cheap LED is suitable for conventional absorbance or fluorescence measurements in the visible range. On the other hand, LEDs-UV (i.e. $<350 \mathrm{~nm}$ ) are more expensive, mainly the recently developed deep-UV LEDs (i.e. $<300 \mathrm{~nm}$ ), which can reach up to 700 USD whereas a LED-UV emitting in the range from 380 to $325 \mathrm{~nm}$ can be found for less than 20 USD. The options for LEDs-IR are available in the range of $900 \mathrm{~nm}-4.5 \mu \mathrm{m}$ with prices generally ranging from 20 to 300 USD. Basically, the further the LED distances from the visible range - in both directions - the more expensive are the costs. For detection in the visible/near-infrared range, Si-based photodiodes are the best option, whereas UV detection can be performed using $\mathrm{SiC}, \mathrm{GaN}, \mathrm{GaP}$, or $\mathrm{TiO}_{2}$ photodiodes. For the IR-range, photodiodes based on InGaAs, InAs, InSb or $\mathrm{HgCdTe}$ are suitable options. Unmounted Si photodiodes can be found in the range of 2 to 200 USD, with differences in spectral sensitivities, active area, dark current, and package type. GaP and InGaAs photodiodes are found for a minimum of 150 USD. All the prices informed here were obtained from Thorlabs supplier website (www.thorlabs.com), excepting conventional visible LEDs and some photodiodes.

As mentioned, many reviews discussing the theoretical aspects of LEDs and detectors devices are available in the literature. Additionally, some interesting works demonstrate the construction of LED-based photometers as an instructional tool for electronic and instrumentation courses using Arduino-based devices. ${ }^{17-23}$ Herein, we have highlighted the most important aspects of each step to assembling a LED-based photometer and fluorometer. Moreover, in this paper, we intend to propose a practical tutorial organized in steps to enable readers to build their photometer of fluorometer with low budget yet providing reliable and satisfactory results showing that this sort of instrument can be used either in research or educational purposes. We believe it is particularly important in the current scenario where there is an increase of cuts towards scientific funding.

\section{EXPERIMENTAL}

\section{Chemicals}

Methyl orange, Potassium indigo trissulfonate, and rhodamine B were obtained by Sigma-Aldrich (USA). Aqueous solutions of the dyes were prepared using Milli-Q deionized water (Millipore, USA). Rhodamine B solutions were prepared using methanol (Vetec, Brazil). 


\section{Electronics}

Different colored LEDs, resistors, and capacitors were acquired from a local electronic store in Uberlândia, Brazil. Si photodiode with photosensitive area of $1.2 \mathrm{~mm}^{2}$ (S12028, Hamamatsu, Japan) was employed as light detector. Operational amplifiers models TLC071CP and LOG101 were obtained from Texas Instrument (USA). A fused deposit modeling-based 3D printer (model A2 Core, GTMax, Americana, Brazil) was used to fabricate 3D structures using ABS thermoplastic filaments.

Arduino UNO was employed to build a microcontroller-based photometer connected to a 16-bit light-to-digital converter sensor (TSL2591 High dynamic range digital light sensor, Adafruit ${ }^{\circledR}$, New York City, USA). Conventional absorbance and fluorescence measurements were performed using a spectrophotometer (Model UV-1800, Shimadzu, Japan) and a spectrofluorometer (Model RF-1501, Shimadzu, Japan). LED spectra were measured using a fiber-optic USB spectrometer (USB4000, Ocean Optics, USA) with Spectra Suite software package.

\section{Power supply}

Generally, integrated circuits (ICs) need to be powered by a dual polarity voltage source (e.g. $+15 \mathrm{~V}$ and $-15 \mathrm{~V}$ ). Herein, the power supply was obtained as follows: A voltage transformer model Trafo ${ }^{24}$ (MS, Brazil) was used to convert input power of 110 or $220 \mathrm{~V}$ to three outputs of $+15,0$, and $+15 \mathrm{~V}$ with a maximum current of $500 \mathrm{~mA}$. Next to it, each transformer output was attached to a suitable electronic circuitry based on the usage of the ICs LM7815 25 and LM7915 ${ }^{26}$ to deliver at the output $+15 \mathrm{~V}, 0$ (Ground), and $-15 \mathrm{~V}$. The circuitry can be assembled in the lab with the aid of a breadboard or a printed circuit board (PCB) that can be bought from online sellers.

\section{Assembling of electronics and measuring tool}

A breadboard is one of the most useful equipment for learning electronics and it consists of a didactic board composed of a matrix of contacts that allows the construction of experimental circuits without the need to weld the components by inserting or removing the components (e.g. resistors, capacitors and wires) quickly and safely. There are several sizes and types of breadboards and usually, they are composed of several horizontal lines that are electrically connected by metal electrical contacts (located in the prototyping area). Thus, each horizontal row of holes is interconnected. The rows can be separated by a central divider, which can create two different groups of non-connected rows. In addition to the horizontal rows, there are long conductive bands that run vertically along the sides (power rail), which are normally used to plug the power supply wires cables. Figure S3 represents a typical breadboard and its internal connections. Additionally, all electrical circuits described in the work are represented as a breadboard-mounted device in the supplementary material.

A multimeter is a simple and yet essential instrument that is useful not only for measuring electrical proprieties but also for electrical and electronic circuits testing. For the development of LED-based instruments, the usage of a multimeter is essential as these measurements involve the encoding of chemical information into electrical signals. Multimeters enable measurements such as electrical voltages (Alternate or constant V, Volts)), current intensity (AC/DC, Amperes), and electrical resistance (R, Ohm). Modern multimeters also allow measurements of capacitance, inductance, frequency, temperature, among others. Analog multimeters can be found, which have a reading display composed of a pointer indicator on a graduated scale, and digital ones, where the reading is indicated on an LCD or LED display. The approach of using the multimeter, regardless of whether it is digital or analog, is similar, as long as you understand its basic reading principle. A multimeter has two probes, one red and one black. The black must be connected to the point on the multimeter indicated with GND or COM (this is called ground or common). The red probe can be connected to two other inputs, but for most measurements, the connection is made at the point indicated as V (voltage), $\Omega$ (Resistance), or $\mathrm{mA}$ (current). The reader can find a discussion about each operation mode in the supplementary information.

\section{D printed platform for absorbance and fluorescence measurements}

The 3D structure is drawn in the CAD software with the desired dimensions and the final version is exported as .stf extension. This file is opened in a slicer software to translate the .stl file into instructions for the 3D printer to follow. Slicing is dividing or chopping the $3 \mathrm{D}$ model into hundreds or thousands of horizontal layers, telling the machine exactly what to do, step by step. After this process, the software will create a new format called G-code (gcode). Then, the $3 \mathrm{D}$ printer can start the printing process.

The 3D structures tailored to hold the optoelectronic devices (i.e. LED and Photodiodes) and the sample cuvette for absorbance and fluorescence measurements were designed using a cad software (Autodesk Inventor 2019) and fabricated by a fused-deposit modelling 3D printer (model A2 Core, GTMax, Brazil). Educational versions of the Autodesk Inventor can be downloaded for free on the homepage (www.autodesk.com.br). Herein, the device was comprised of a rectangular piece with external dimensions of $25 \times 25 \times 45 \mathrm{~mm}$ (L x H x h). A $10 \mathrm{~mm}$ quartz or plastic cuvette was inserted in a rectangular hole into the device with dimensions of $11 \times 11 \times 4 \mathrm{~mm}(\mathrm{~L} \times \mathrm{H} \mathrm{x} \mathrm{h})$. A hole was designed in one of the rectangular faces with a diameter of $5 \mathrm{~mm}$ to positioning the LED as the light source. For absorbance measurements, the photodiode was positioned directly on the opposite side of the LED (Figure 2a) and, for fluorescence measurement, the photodiode was positioned in the $90^{\circ}$ face (Figure $2 \mathrm{~b}$ ) to avoid the influence of the LED as an excitation light source. The main printing conditions are described in the supplementary information and the overall costs were less than 5 USD. Table 1 shows an estimation of average cost of each device.

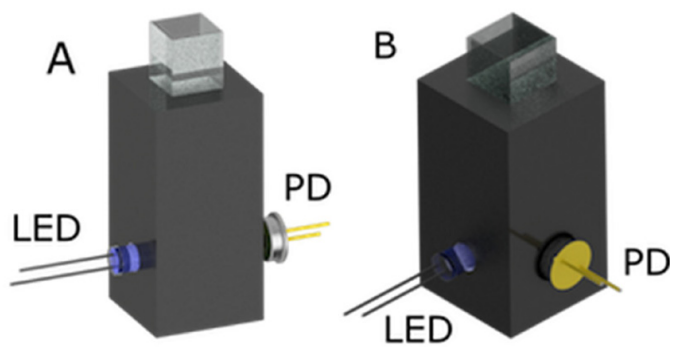

Figure 2. CAD drawing representation of the $3 D$ printed device for (a) absorbance measurements and (b) fluorescence measurements using a LED/ PD based device

\section{RESULTS AND DISCUSSION}

\section{Absorbance measurements}

\section{Step 1. Picking the most suitable LED}

The Beer-Lambert law states that the absorbance (A) has a linear relation with the analyte concentration and can be indirectly obtained from measuring the light intensities before $\left(\mathrm{I}_{0}\right)$ and after $(\mathrm{I})$ passing through the absorbing medium (b) (i.e. optical pathlength). 
Table 1. Information on average costs estimation for each device

\begin{tabular}{|c|c|c|c|c|c|}
\hline & LED & Photodiode & 3D structure & Electronics & Total \\
\hline UV-LED device & $\begin{array}{c}350 \text { USD (Deep-UV) } \\
15 \text { USD (UV) }\end{array}$ & 200 USD & 5 USD & 10 USD & $\begin{array}{c}565 \text { USD (deep-UV) } \\
230 \text { USD (UV) }\end{array}$ \\
\hline VIS-LED device & 1 USD & 25 USD & 5 USD & 10 USD & 41 USD \\
\hline $\begin{array}{l}\text { USB spectrometer + } \\
\text { Lamp + Fibers }\end{array}$ & & & & & 5,000 USD \\
\hline
\end{tabular}

If light intensities are measured with photodiodes operating in the photocurrent mode absorbance can be obtained from using current after $\left(i_{0}\right)$ and before (i) interaction with the absorbing medium (where $i_{D}$ is the dark current). The relation is equally true to the Voltage instead of the current if using a current-to-voltage converter. Equation 1 shows the described relation.

$$
A=-\log \frac{I}{I_{0}}=-\log \frac{i-i_{D}}{i_{0}-i_{D}}=\varepsilon b C
$$

where: $\varepsilon=$ molar absorptivity coefficient.

Strictly speaking, Beer-Lambert law is theoretically true only for monochromatic radiation. However, even a commercial spectrophotometer does not provide monochromatic light, as it has 2-10 nm bandwidth. Broader bandwidth may lead to spectral distortion. LEDs have a typical FWHM of 10-30 nm and therefore can be considered a non-monochromatic radiation source. In such a case, the Lambert-Beer law is modified by taking account of the variation of the intensity of the light source $\left(\mathrm{I}_{0}\right)$, the sensitivity of the detector $(\mathrm{S})$, and the molar absorptivity across $(\varepsilon)$ the band of the light source, according to the equation $2 .{ }^{6}$

$$
A=-\log \frac{\int_{\lambda_{0}}^{\lambda_{1}} S(\lambda) \cdot I_{0}(\lambda) \cdot 10^{-\varepsilon(\lambda) b C} d \lambda}{\int_{\lambda_{0}}^{\lambda_{1}} S(\lambda) \cdot I_{0}(\lambda) d \lambda}
$$

Therefore, the broad behavior of the emission spectra of LED can lead to non-linear calibration curves and also results in high limits of detection (LODs). In practical terms, the deviation of linearity is higher when the absorbance bands of the analyte are not well matched with the LED emission spectra. Therefore, picking the right LED which means matching the peak emission wavelength of LED to the analyte absorption maximum - is the most crucial part to achieve suitable linearity and low LODs. Figure 3 describes good and poor examples of LED selection for the development of an analytical method based on absorbance measurements.

\section{Step 2. Driving a LED}

A typical $5 \mathrm{~mm}$ light-emitting diode has two legs related to the polarity (positive (anode) and negative (cathode)). Any diode only allows current to flow in one direction. It means they will only light up if you connect the legs to the power supply properly. The long leg is the anode, which must be connected to the power supply, and the short leg (flat side of the LED) goes to the ground (GND. Fluctuations in the voltage of the LED produces instability of the output light intensity and can even cause the failure of the device. Thus, LEDs must be driven with a constant current source (CCS) and not directly connected to a power supply even if using a resistor to limit the current. CCS can be easily obtained by designing an appropriate electric circuitry based on e.g. the usage of a voltage regulator integrated circuit (IC) - such as the LM317 27 - and ordinaries capacitors and resistors. A suitable configuration of a CCS circuitry based on LM317 is shown in Figure S4. Each pin of the LM317 (i.e. Input (I), Output (O), and

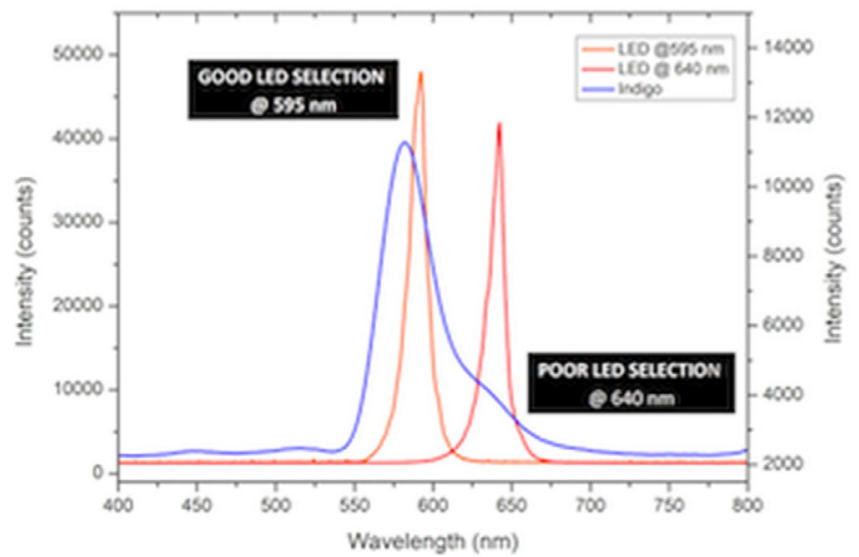

Figure 3. Representation of the emission spectra of two LEDs (595 and 640 $n m$ ) and the absorption spectrum of an indigo solution

Adjust (A)) must be correctly connected in order to provide constant current, otherwise, it may cause damage to the LED. The $+15 \mathrm{~V}$ and GND outputs of the power supply can be used to provide voltage and current to the circuitry. Note that the current which will feed the LED is limited and controlled by the resistor $\mathrm{R}$ according to equation 3 . In operation, the LM317 develops a nominal $1.25 \mathrm{~V}$ reference voltage between the output $(\mathrm{O})$ and adjustment $(\mathrm{A})$ terminal. ${ }^{23}$

$$
R=\frac{1.25}{i}
$$

Each LED has its particular current specifications, which can be found in the product datasheet and it must be checked in order to calculate an appropriate resistor. Generally, the maximum DC forward current of visible LED is in the range of $20-30 \mathrm{~mA}$, whereas deep-UV and IR LEDs have higher ratings. By using equation 3 it is possible to calculate the appropriate resistor $\mathrm{R}$ which must be used in the LED circuitry. An interesting alternative is to employ a variable resistor (e.g. potentiometer) to increase or decrease the light intensity of the LED by changing the DC current produced by the circuitry. An important hint is testing the circuitry by measuring the current before attaching the LED, in order to avoid errors and damage to the components. Deep-UV LEDs require additional care due to the increase of the temperature of the diode, leading to changes in the light emission intensity and also the possibility of failure. Heat sinkers are advised to be employed for thermal dissipation.

\section{Step 3. Operating the photodiode as a light detector}

\section{Transmittance measurements}

Light detection should be performed using a suitable photodiode with appreciable responsivity in the spectral region of the lightemitting diode. For example, Si-based PDs are the best option for visible radiation. Photodiodes must be correctly operated to achieve suitable performance with low-noise, fast response, and precision. 
Fundamentally, photodiodes can be operated to provide electrical signals in different modes, such as photocurrent (i.e. current measurements) or photovoltaic mode (i.e. voltage measurements). For quantitative purposes, the photocurrent mode is often preferred as the light intensity is proportional to the current generated at the photodiode and has superior precision than the photovoltaic mode. In order to measure the photocurrent, it is necessary to employ an operational amplifier (Op Amp) - such as TLC071, CA3140 and OP07 - connected to the photodiode. Figure S5 shows all the connections that must be made to operate a photodiode using the TLC071, ${ }^{28}$ however, there are many other operational amplifiers available in the market. Each op-amp has its own resistor and capacitor relationship and the user must be mindful of the pin configurations of each IC available in the datasheet and must be checked.

In this configuration, the operational amplifier acts as a currentto-voltage converter, therefore, the output voltage (Vo) measured in this circuitry is proportional to the light intensity that reaches the detector. It is important to note that the signal obtained in this arrange is equivalent to transmittance measurements and, therefore, not linear to the analyte concentration. In such cases, the operator must measure the transmittance of the blank $\left(\mathrm{I}_{0}\right)$ before measuring the analyte transmittance (I) and convert the values to absorbance by applying the logarithm relation between $\mathrm{I}_{0}$ and $\mathrm{I}$.

\section{Absorbance measurements}

The output voltage obtained from photodiodes can be directly related to absorbance by using logarithms ratio amplifiers rather than conventional operational amplifiers. Log ratio amplifiers - such as LOG101 from Texas Instrument ${ }^{29}$ - are a versatile integrated circuit that computes the logarithm or log ratio of an input current relative to a reference current. By using one photodiode attached to the signal input and one photodiode attached to the reference input, the output voltage $\left(\mathrm{V}_{0}\right)$ can be directly related to absorbance as $1 \mathrm{~V}=1 \mathrm{AU}$. An example of a log ratio amplifier circuitry-based is shown in Figure S6. It is important to note that LOG101 is only available in a surface mount package (SMD), which means that it cannot be used directly on a breadboard. However, it is possible to employ SOIC adaptors (e.g. SOIC-8) to enable the connection of a SMD component onto a breadboard. Additionally, in case of LOG 101 is not available, the absorbance can be obtained mathematically from the transmittance measurements.

The employment of a reference photodiode brings additional complexity to the design of the photometer, as the light emitted by the LED must be divided by using a beam splitter towards the reference PD. This approach is used to compensate temperature fluctuations of the LED which can affect the light intensity emission, particularly for deep-UV LEDs. However, an alternative approach can be performed by attaching the reference input to a current source, providing a reference signal which will be used by the log ratio amplifier rather than the current generated by a photodiode. Additionally, the output voltage can be processed with an offset circuitry based on an operational amplifier to compensate for an imbalance between the intensities of the two signals. Despite this setup is not mandatory, it is very useful to zero absorbance reading using a blank solution before the measurements.

\section{Step 4. Performing absorbance measurements}

After picking the appropriate LED and assembling the electric circuitry to drive the LED properly and obtain the voltage output from the photodiode related to either transmittance or absorbance, the user is prepared to perform the measurements. Please note that LED and photodiode(s) must be wired to the integrated circuits and resistors located in the breadboard. A structure designed and fabricated by a
$3 \mathrm{D}$ printer is used to housing the LED, photodiode, and a conventional cuvette, as described in the experimental section. The usage of a 3D printer to build optical instrumentation is not mandatory, however, the easiness of use, low-cost and fastness of the printed structures by additive manufacturing is highly attractive. The output voltage was measured by using a digital multimeter in the voltage mode. In order to evaluate the performance of the proposed photometer, we have performed two different experiments

\section{(a) Comparing analytical performance between LED photometer and spectrophotometer}

The analytical performance of the developed LED photometer can be demonstrated by comparing the results with those obtained by a conventional spectrophotometer. For this, methyl orange dye was selected as an analyte to evaluate the designed analytical figure-of-merits. A LED emitting at $460 \mathrm{~nm}$ was selected and the absorbance was obtained by a log-ratio amplifier circuitry (Figure S5). Solutions in the range of 2.5 to $16 \mu \mathrm{mol} \mathrm{\textrm {L } ^ { - 1 }}$ were prepared and the absorbance was measured using a plastic cuvette with an optical pathlength of $10 \mathrm{~mm}$. Figure 4 shows the analytical curves obtained by the LED photometer and the conventional spectrophotometer and table 2 the values of each analytical curve.

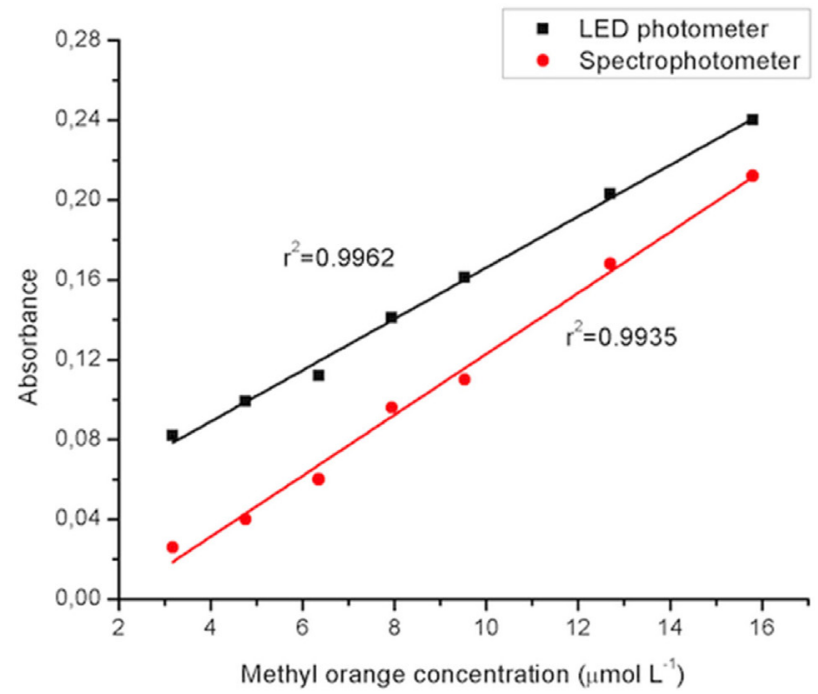

Figure 4. Analytical curves obtained using the LED-based photometer and a conventional spectrophotometer

Table 2. Figure-of-merits of the analytical curves build using the LED-based photometer and a conventional spectrophotometer

\begin{tabular}{lcc}
\hline & LED Photometer & Spectrophotometer \\
\hline Slope & 0.01285 & 0.01526 \\
Intercept & 0.03769 & -0.02960 \\
Correlation coefficient & 0.9962 & 0.9935 \\
Linear range $\left.(\mu \mathrm{mol} \mathrm{L})^{-1}\right)$ & $2.5-16$ & $2.5-16$ \\
RSD (blank, $\mathrm{n}=5)$ & $5 \%$ & $1.5 \%$ \\
LOD $(\mu \mathrm{mol} \mathrm{L-1)}$ & 0.12 & 0.08 \\
\hline
\end{tabular}

As can be demonstrated by table 2, the analytical performance of the LED-based photometer is similar to a conventional spectrophotometer in terms of sensitivity and linearity. The spectrophotometer has a Si-photodiode as a detector element. It is important to note that the circuitry was assembled on a breadboard with exposed wires, which certainly increased the noise effect. For precise measurements, the circuitry must be mounted using a 
grounded box (if made of metal) containing the printed circuit board (PCB) and the wires. Also, the suitable linearity of the LED-based device was obtained due to the match between the LED emitting band and the absorption band of the analyte.

\section{(b) Comparing the analytical performance of two LEDs}

In some cases, there is no exact match between one light-emitting diode spectra with the absorption band of the analyte. For instance, the absorbance spectra of the red complex nickel (II) dimethylglioximate (max absorption at $540 \mathrm{~nm}$ ) does not match exactly with any available LED, and rather two possibilities are available with maximum emission at 525 or $565 \mathrm{~nm}$. In this scenario, the linearity of each measurement must be evaluated to obtain the best performance. Here, we have prepared solutions of different concentrations of nickel (II) dimethylglioximate and it was obtained analytical curves for absorbance measurements for each LED for comparison purposes. As can be seen in Figure 5, the more suitable LED for this example is the one emitting at $525 \mathrm{~nm}$.

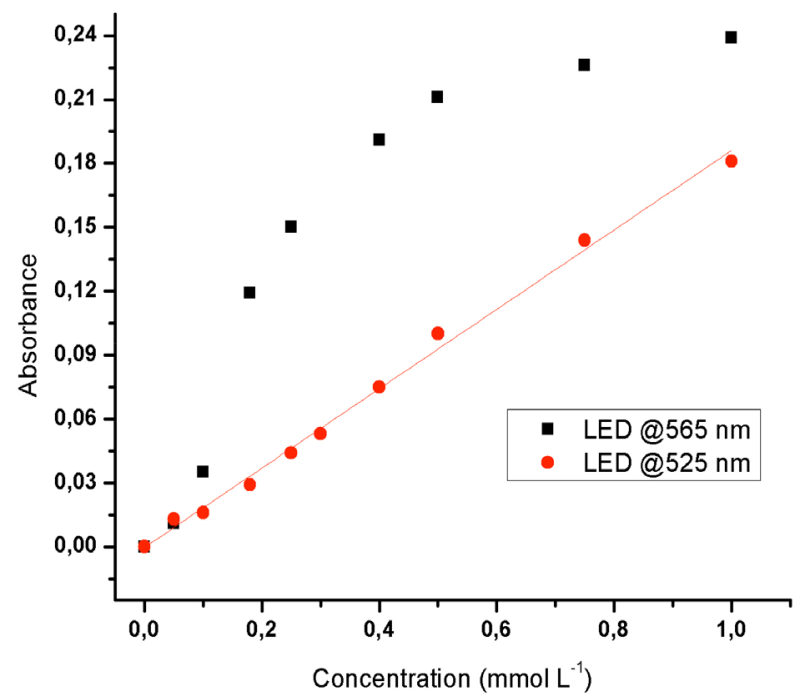

Figure 5. Analytical curves obtained by measurements of nickel (II) dimethylglioximate absorbance using LEDs emitting at different wavelengths

\section{Fluorescence measurements}

The fluorescence intensity (FI) can also be linearly related to the analyte concentration as demonstrated in equation 4 .

$$
F I=K^{\prime}\left(P_{0}-P\right)
$$

where $\mathrm{P}_{0}$ is the radiant power of the incident light, and $\mathrm{P}$ the power after it passes thought a pathlength (b), and $\mathrm{K}^{\prime}$ is a constant that depends on the quantum efficiency of the fluorophore. The fluorescence intensity varies linearly with the path length, the analyte concentration and to the intensity of the incident radiation, according to equation 5 .

$$
F I=2.303 K \varepsilon b c P_{0}
$$

where $\varepsilon$ is the molar absorptivity. If the optical pathlength and the intensity of the light source are kept constant, the fluorescence intensity can be used for quantitative purposes. In the next steps, we will demonstrate how to construct and apply a LED-based fluorometer.

Step 1. Selecting and operating LED as a light excitation source

LEDs can be used for molecular fluorescence experiments as light excitation sources and photodiodes are employed in the photocurrent mode to measure the light intensity emitted by the fluorophore. In this case, the fluorescence is directly proportional to the intensity of the excitation light source, which means that a lower limit of detection can be reached with high power LEDs (Equation 5). However, the majority of fluorescent compounds are excited at shorter wavelengths, such as ultraviolet and blue light, while few fluorophores are excited in the green (e.g. fluorescein and rhodamine) region. Despite LEDs emitting at ultraviolet have generally low optical power, lower limits of detection can be overall reached in fluorescence than absorbance measurements. In order to select the appropriate LED, the excitation spectrum of the fluorophore must match with the LED emitting spectrum, similar to the absorption mode. Herein, we have selected Rhodamine B as fluorophore $\left(\lambda_{\mathrm{Ex}}=525 \mathrm{~nm}\right)$, and figure 6a shows the emission of Rhodamine B when excited with LED @ $525 \mathrm{~nm}$.

The LED can be driven in the same LM317-based electric circuitry used for absorbance measurements. As mentioned, increasing the light intensity will promote higher fluorescence intensity (Figure 6b) and therefore lower limit of detection, however, it is important to note that higher excitation light can reach the detector and enhance the background noise of the measurement. Therefore, it is essential to find and fix the right balance between high fluorescence intensity and low background signal before performing the calibration. Another important aspect regards the position of the LED and the photodiode. Similarly, to conventional a spectrofluorometer, the excitation source (i.e. LED) must be positioned $90^{\circ}$ from the detector (i.e. photodiode), in order to avoid light interference from the source. It is important to mention that for ideal fluorescence measurements, the intensity of the emitted light should be higher than the incident light from the LED. Otherwise, light scattering effects can be observed in the
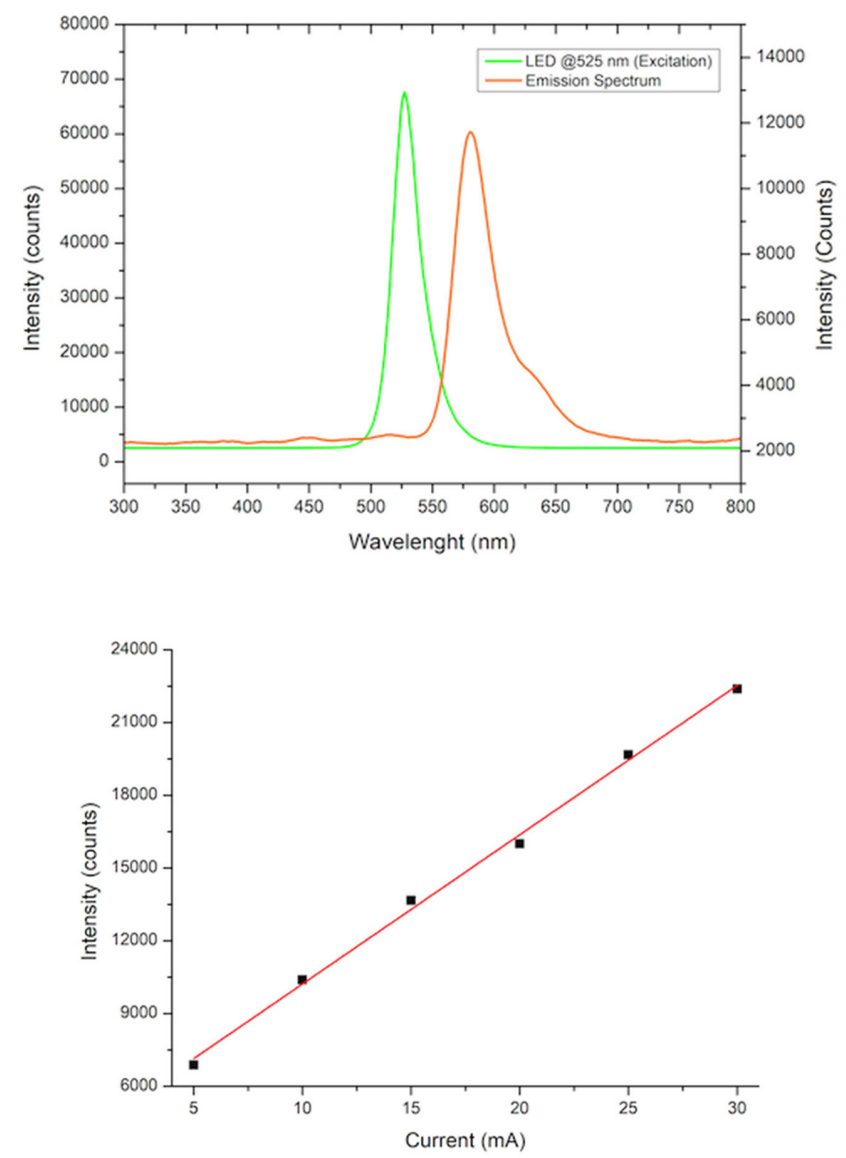

Figure 6. (a) Emission spectrum of Rhodamine B when excited with a LED @ $525 \mathrm{~nm}$ (green line) and (b) influence of the LED driven current in the fluorescence intensity. Rhodamine concentration: $5 \mu \mathrm{mol} \mathrm{L} \mathrm{L}^{-1}$ 
detector. The usage of suitable filters to minimize the incidence of the excitation light is also fundamental to refine the signal related to the emitted light.

\section{Step 2. Measuring light intensity}

The emitted light from the fluorophore after excitation from the LED emission can be detected using a conventional photodiode usually, a Si photodiode is suitable as fluorescence generally occurs in the visible range - appropriately operated in the photocurrent mode. Operational amplifiers (e.g. TLC071CP) can be employed to convert the current generated in the PD to voltage, similarly to the transmittance measurements setup described in the previous section, which is proportional to concentration. The complete electronic circuitry is exemplified in Figure S5.

\section{Step 3. Performing fluorescence measurements}

After the assembling of the LED-based fluorometer, the digital multimeter is employed to perform voltage measurements, which is linear to the light intensity and therefore to the analyte concentration. In order to demonstrate and evaluate the performance of the device (Figure S2), rhodamine B was selected as fluorophore with concentration in the range of 0.4 to $2.5 \mu \mathrm{mol} \mathrm{L^{-1 }}$. A longpass filter (FEL550, Thorlabs, United States) was positioned between the cuvette and the photodiode to minimize the interference of the incident light. A LED emitting at $525 \mathrm{~nm}$ with a current at $30 \mathrm{~mA}$ was employed as an excitation source. Table 3 shows the figure-of-merits of the analytical curves obtained by the LED-based fluor0meter and a conventional spectrofluorometer.

Table 3. Figure-of-merits of the analytical curves build using the LED-based fluorometer and a conventional spectrofluorometer with photomultiplier tube detector

\begin{tabular}{lcc}
\hline & LED Fluorometer & Spectrofluorometer \\
\hline Slope & 2.11 & 5.24 \\
Intercept & 0.90 & 0.88 \\
Correlation coefficient & 0.991 & 0.997 \\
RSD $(\%)(\mathrm{n}=5)$ & 4.9 & 0.8 \\
LOD & 0.10 & 0.008 \\
\hline
\end{tabular}

\section{Attaching a light-to-digital converter sensor to a microcontroller for light intensity measurements.}

Arduino is an open-source microcontroller board that consists of a printed circuit board based on the ATmega328 microprocessor with a variety of input/output pins that can easily be interfaced with other expanded boards. Originally, the Arduino board was developed by Arduino LLC (Ivrea, Italy) to be a device for beginners on electronics and software programmers. Cheap and affordable ( 23 USD), this microcontroller is an excellent alternative for measuring and processing electrical signals generated by a variety of sensors (e.g. light, temperature, pressure). Usually, these sensors convert the physical propriety to the analog output signal (e.g. voltage), which will be processed by the Arduino through attaching the sensor to the analog inputs of the board. There are some interesting works in the literature demonstrating the usage of an Arduino-based platform for the development of low-cost instruments for educational purposes. ${ }^{17,18}$ For light intensity measurements, the use of light-todigital converters embedded with a photodiode - such as TSL2591 $(\sim 7 \mathrm{USD})-$ is an alternative to develop a LED-based photometer or fluorometer. An integration analogic-to-digital (ADCs) convert the photodiode currents into a digital output that represents the irradiance measured. Luminosity sensors is calibrated digital light sensor IC that measures the incident light intensity and converts it into a 16-bit digital number. The measured light level is displayed in Lux units, a SI (International System of Units) unit for illuminance and is equal to one lumen per square meter. These signals are directly used for absorbance calculations.

A descriptive guide for the connection of the light-to-digital sensor to the Arduino Uno board is described in the supplementary information section. In this configuration, the calibrated light-to-digital converter is attached to an Arduino board via the input channel. An analogic-todigital IC (ADCs) converts the photodiode currents generated by the light into a digital output that represents the irradiance measured. The incident light intensity (I) is then converted into a high-resolution 16-bit digital data and expressed in a computer running the Arduino software (Arduino IDE). The measured light level is displayed in Lux units, a SI (International System of Units) unit for illuminance, and is equal to one lumen per square meter. These signals are used for transmittance of fluorescence measurements, as demonstrated in the previous sections. For absorbance measurements, the user must measure first the light intensity of the blank solution followed by the sample solution. The logarithm ratio is then related to absorbance as demonstrated in Equation 2. An Arduino-based photometer/fluorometer is advantageous when the analytical signal must be measured and stored in function of time, enabling real-time monitoring of the light intensity. Figure S7 shows the Arduino-based circuitry for the usage of the light-to-digital converter and a step-by-step instruction.

\section{CONCLUSIONS}

Analytical methods based on the measurement of absorbance and fluorescence are a powerful tool in the analytical chemistry field. Conventional benchtop instruments such as spectrophotometers and spectrofluorometers are essential for research development in the analytical as well to many other fields, including for teaching purposes. Despite the costs of such instruments are inferior when compared to other techniques (e.g. HPLC, IR, MNR, MS), there are still many research groups and universities which cannot afford the prices, especially when public politics continuously withdraw science funding. Here, we have shown that is possible to build a setup to perform absorbance and fluorescence measurements using conventional LEDs, some optoelectronics, and a 3D printer, which has an affordable price. Besides, we have demonstrated that the analytical performance of the LED-based device is comparable to a conventional spectrophotometer and a spectrofluorometer. Many chemists are not familiar with electronics and the development of "lab-made" devices may appear as an unfeasible challenge. Therefore, we expect that this tutorial can act as a guide and enable the construction of absorbance/ fluorescence-based devices.

\section{SUPPLEMENTARY INFORMATION}

Electronic supplementary information (ESI) available free of charge at quimicanova.sbq.org.br.

\section{ACKNOWLEDGMENTS}

The authors are thankful for the financial support provided by the Brazilian National Council for Scientific and Technological Development (CNPq, Proc. 428094/2018-0, Proc. 425114/2018-0 and Proc. 425494/2018-7) the Coordination of the Improvement of Higher Education (CAPES), and the state governmental agency 'Fundação de Amparo à Pesquisa do Estado de Minas Gerais' (FAPEMIG, Proc. APQ-02272-18). 


\section{REFERENCES}

1. Skoog, D.A; West, D.M.; Holler, F.J.; Crouch, S.R. Fundamentals of analytical chemistry, $9^{\text {th }}$ edition, Cengage Learning, Inc., 2013

2. Fortes, P. R.; da Silveira Petruci, J. F.; Raimundo, I. M.; Optical Gas Sensors for Exhaled Breath Analysis; 2017.

3. Gouda, A. A.; Shafey, Z. El; Hossny, N.; El-Azzazy, R.; Spectrochim. Acta - Part A Mol. Biomol. Spectrosc. 2008, 70, 785.

4. Rocha, C. O.; Petruci, J. F. S.; Cardoso, A. A.; J. Braz. Chem. Soc. 2017, 28, 1528-1523.

5. Fernandes, G.M.; Silva W.R; Barreto, D.N,; Lamarca, R.S., Gomes, P.C.F.L., Petruci, J.F.S., Batista, A.D. Anal. Chim. Acta. 2020, 1135:187.

6. Bui, D. A.; Hauser, P. C.; Anal. Chim. Acta 2015, 853, 46.

7. Flaschka, H.; McKeithan, C.; Barnes, R.; Anal. Lett. 1973, 6, 585.

8. Anfält, T.; Granéli, A.; Strandberg, M.; Anal. Chem. 1976, 48, 357.

9. Hauser, P. C.; Tan, S. S. S.; Analyst 1993, 118, 991.

10. Petruci, J. F.S; Liebetanz, M. G.; Cardoso, A. A.; Hauser, P. C.; J. Chromatogr. A 2017, 1512, 143.

11. Schmid, S.; Macka, M.; Hauser, P. C.; Analyst 2008, 133, 465.

12. Bui, D. A.; Hauser, P. C.; J. Chromatogr. A 2015, 1421, 203.

13. Macka, M.; Piasecki, T.; Dasgupta, P. K.; Annu. Rev. Anal. Chem. 2014, 7, 183.

14. Dasgupta, P. K.; Eom, I. Y.; Morris, K. J.; Li, J.; Anal. Chim. Acta 2003, $500,337$.

15. O’Toole, M.; Diamond, D.; Sensors 2008, 8, 2453.
16. Dasgupta, P. K.; Bellamy, H. S.; Liu, H.; Lopez, J. L.; Loree, E. L.; Morris, K.; Petersen, K.; Mir, K. a; Talanta 1993, 40, 53.

17. McClain, R. L.; J. Chem. Educ. 2014, 91, 747.

18. Kubínová, Š.; Šlégr, J.; J. Chem. Educ. 2015, 92, 1751.

19. Bougot-Robin, K.; Paget, J.; Atkins, S. C.; Edel, J. B.; J. Chem. Educ. 2016, 93, 1232.

20. Fisher, D. K. \& Gould, P. J. Mod. Instrum. 2012, 01, 8.

21. Soares, F. S. C; Vieira, A.L.; Soares, M.S.; Sant'anna, M.V.; Nascentes, R.; Bossu, C.M. Quim. Nova 2018, 41, 1196.

22. De Morais, C.L.; Carvalo, J.C.; Sant'Anna, C.; Eugenio, M.; Gasparotto, L.H.S.; Lima, K.M.G. Anal. Methods 2015, 7, 7917.

23. Zinner, K; Shimizu, Y. Quimica Nova 1978, 1, 38.

24. https://www.filipeflop.com/produto/transformador-trafo-15v-500mabivolt/. Acessed in September 2020

25. https://www.mouser.com/datasheet/2/308/LM7815-1120980.pdf. Acessed in September 2020.

26. ht tps://www.ti.com/lit/ds/s nosbq 7 c/s nosbq 7 c. pdf?ts=1599500063737\&ref_url=https\%253A\%252F\%252Fwww. google.com\%252F. Acessed in September 2020.

27. https://www.ti.com/document-viewer/LM317/datasheet/featuressces1289655\#SCES1289655. Acessed in September 2020.

28. https://www.ti.com/product/TLC071. Acessed in September 2020.

29. https://www.ti.com/lit/ds/symlink/log101.pdf?ts=1599500311771. Acessed in September 2020. 